ENCYCLOPÉDIE Encyclopédie berbère

BERBERE $\quad 30 \mid \mathbf{2 0 1 0}$

30 | Maaziz - Matmata

\title{
Maktar, Makthar / Mactaris
}

\section{Ghaki}

\section{OpenEdition}

Journals

Édition électronique

URL : http://journals.openedition.org/encyclopedieberbere/433

DOI : $10.4000 /$ encyclopedieberbere.433

ISSN : 2262-7197

\section{Éditeur}

Peeters Publishers

\section{Édition imprimée}

Date de publication : 29 décembre 2010

Pagination : 4535-4540

ISBN : 978-90-429-2367-6

ISSN : 1015-7344

Référence électronique

M. Ghaki, « Maktar, Makthar / Mactaris », Encyclopédie berbère [En ligne], 30 | 2010, document M26, mis en ligne le 22 septembre 2020, consulté le 13 octobre 2020. URL : http://journals.openedition.org/ encyclopedieberbere/433; DOI : https://doi.org/10.4000/encyclopedieberbere.433

Ce document a été généré automatiquement le 13 octobre 2020

(c) Tous droits réservés 


\title{
Maktar, Makthar / Mactaris
}

\author{
M. Ghaki
}

\section{Le nom}

1 Le nom de la cité est demeuré le même depuis l'antiquité même s'il a subi, à travers le temps, de légères altérations: dans les inscriptions néo-puniques collectives, il est question des «ba'lim de MKTRYM»; à l'époque romaine, les nombreuses inscriptions renferment le plus souvent la forme mactaris; dans l'une des listes conciliaires, il est question de Macthari ; aujourd'hui, le nom dans sa forme arabisée est Makthar.

2 Makthar se trouve dans le Haut Tell, à quelques $160 \mathrm{~km}$ de Tunis vers le centre-ouest. Elle se caractérise par sa localisation en altitude, à quelques 1.000 mètres, un climat continental et une région agricole riche.

\section{Makthar la numide}

3 Le site de Makthar est occupé depuis la Préhistoire. La région se caractérise par la présence de grandes nécropoles mégalithiques et par une architecture funéraire unique ; il n'y a nulle part ailleurs des tombes ayant la forme des tombes mégalithiques de Makthar, de Hammam ez-Zouakra, d'Ellès ou de Kbor el-Ghoul. La région est, déjà au II siècle avant J.C., fortement urbanisée : citons à titre d'exemples Mididi, Hammam Zouakra, Uzappa, Kisra, Maghraoua-Macota*; la liste est bien plus longue en réalité puisque l'épigraphie latine parle de plus de soixante cités constituant les deux pagi Tuscae et Gunzuzi.

4 La croissance de la cité semble s'être faite petit à petit et l'on ne peut parler d'une ville appelée Makthar qu'à l'époque des rois numides. Au cours du IV siècle avant J.C., les échanges commerciaux mais aussi humains avec Carthage et le littoral phénicopunique entraînent une dynamique chez les Numides: naissance de centres marchés pour les échanges de marchandises et pour le recrutement de mercenaires; ces noyaux vont se développer jusqu'à devenir des centres urbains. 
Makthar numide est caractérisée par les aspects suivants :

- L'épigraphie libyque. la collection des textes funéraires libyques est relativement riche; citons pour mémoire la fameuse bilingue libyque/néo-punique publiée par Chabot sous le numéro 31.

- Les structures mégalithiques: elles sont aujourd'hui au nombre de 25. À observer avec attention les vestiges d'époque romaine, on remarque que l'une des pierres utilisée pour édifier les mégalithes - le grès coquillier - se rencontre parfois sous forme de grandes dalles réemployées, y compris dans le dallage des rues; il est donc fort possible que certains monuments mégalithiques aient fini par disparaître à force de servir de carrières. Leur nombre est aussi trompeur, s'agissant de structures funéraires complexes comptant parfois jusqu'à quatre chambres relativement grandes $-2 \mathrm{~m} \times 2 \mathrm{~m} \times 2,5 \mathrm{~m}$ - et pouvant recevoir des dizaines de morts ; les fouilles menées sur certaines structures confirment aussi bien l'usage répété que la longévité de l'utilisation de ces tombes: il s'agit de structures historiques remontant, au plus tôt, au milieu du premier millénaire; la céramique qui fut découverte dans deux monuments, le mégalithe Pauphilet et le «nouveau mégalithe », se situe entre le quatrième siècle avant J.C. et le premier siècle après J.C. Certains monuments ont donc servi quatre siècles.

- La muraille: dont la partie visible est conservée sur plusieurs dizaines de mètres; elle est d'apparence numide sans qu'elle ait pu être datée avec précision.

- La céramique modelée : les pièces mises au jour suite aux fouilles de structures funéraires mégalithiques se distinguent par une grande variété de formes.

\section{La punicisation}

6 La région de Makthar fait partie du territoire numide même si elle a été annexée par Carthage, probablement au milieu du troisième siècle. Elle demeurera sous administration punique près d'un siècle. Ceci étant, la frontière culturelle est autre : l'influence punique est évidente dès le $\mathrm{V}^{\mathrm{e}}$ siècle J.C. Le résultat de ces contacts est l'introduction des composantes de la civilisation phénico-punique - croyances, rites funéraires, langue, écriture, etc.

Plusieurs monuments puniques furent mis au jour à Makthar et la collection d'objets " puniques » est importante, aussi bien en quantité qu'en valeur historique :

- Le sanctuaire de Baal Hammon: paradoxalement, le monument lui-même n'est toujours pas localisé avec précision.

- Le grand nombre de stèles votives - dont l'inventaire précis n'est encore pas fait: la plupart provient de la zone de l'arc dit de Ras el-'Aïn et des bords sud de l'oued qui traverse la ville. Il s'agit de la troisième collection de stèles votives après celles de Carthage et de Cirta : plus de deux cent textes. Les stèles connues renferment une belle écriture néopunique et un décor souvent riche : disque anthropomorphisé, disque radié, croissant, caducée, etc. Seul Baal Hammon est évoqué dans les formules votives, Tanit demeure inconnue à Makthar.

- Le temple de Hoter Miscar : Hoter Miscar, dont l'étymologie du nom demeure une énigme, est une divinité punique attestée à Carthage où trois inscriptions évoquent ce dieu. Le temple de Hoter Miscar à Makthar a été fouillé par G. C. Picard; cinq textes néo-puniques en proviennent. L'un d'eux évoque le mizrah et donne la liste des personnes membres de cette « confrérie »; il s'agit du plus long texte néopunique connu à ce jour. 
- Les textes funéraires d'époque néopunique illustrent l'évolution de la société makhtaroise et sa punicisation : nombreux sont les défunts qui portent des noms sémitiques mais dont les pères ont des noms libyques.

\section{Makthar la romaine} degré de romanisation atteint par Makthar. L'archéologie - le site n'est fouillé que sur une petite partie - ne permet d'identifier qu'un seul temple, celui d'Apollon attesté par une inscription latine et identifié par Picard; il est un peu décentré par rapport au centre de la ville antique.

Le temple de "Saturne africain ", héritier de Baal Hammon, est encore à découvrir ; une seule inscription latine le mentionne. L'emplacement du temple de Saturne peut très bien recouvrir le sanctuaire de Baal Hammon qui pourrait donc se situer au nordouest de la cité antique, sur la rive droite de l'oued, en amont des endroits où furent découvertes les premières inscriptions votives consacrées au dieu punique.

Les principaux monuments encore visibles de nos jours sont :

- L'arc de Ras el-'Aïn qui marquerait l'entrée nord de la cité ;

- Le monument à auges ;

- Le forum ;

- Le Capitole construit vers 180 après J.C. ; c'est à cette époque que Makthar devient colonie romaine.

-L'agora numide » : il s'agit d'une place dallée située au sud-ouest du Forum; elle doit son appellation à G. C. Picard. Cette place est intégrée dans le tissu urbain de la ville à l'époque romaine ; sa qualification de « numide » pose problème.

- La «Scola des Iuvenes»: Picard définit ce monument, identifié grâce à l'inscription découverte en place et à l'intérieur du monument, comme un espace réunissant des jeunes au sein d'une structure paramilitaire. La salle, partie principale et centrale du monument, servait de lieu de réunion.

- Les thermes sont célèbres pour leur bon état de conservation et pour avoir été transformés en fortification à l'époque byzantine. Ils furent l'objet de fouilles et de restauration. La mosaïque dite « du labyrinthe », impressionnante par sa taille, occupe le sol du gymnase.

- L'arc de Trajan, daté par l'inscription de 116 après J.C.

- Le temple d'Apollon.

- Le mausolée-tour

- L'aqueduc édifié vers 200 après J.C.

\section{Makthar la chrétienne}

Elle est documentée par d'importantes inscriptions funéraires chrétiennes en mosaïques polychromes. Deux monuments païens furent transformés en églises : la «Scola des Iuvenes » et le temple de Hoter Miscar. D'autres lieux de culte chrétien sont connus, dont l'église de Rutilius et la basilique d'Hildequns. Les chrétiens mactarois étaient représentés aux conciles de 256 et de 411, et les épitaphes mises au jour à Makthar même dans les différents monuments chrétiens permettent de dresser une liste d'évêques, prêtres et autres hommes d'église ayant officié à Makthar. Ainsi en 256, il est question de Marcus a Macthari ; à la conférence de 411, Comparator est cité en tant

Encyclopédie berbère, $30 \mid 2010$ 
qu'évêque donatiste, tandis que les «Etats» de l'église africaine de 484 citent un évêque portant le nom de Adelfius, etc.

\section{L'évolution du statut municipal de Makthar}

Plusieurs découvertes permettent d'avoir une certaine idée du statut de la cité et de ses évolutions :

- Des textes datés par la mention de magistrats éponymes portant le titre de suffète. L'originalité réside dans le fait qu'ils sont trois, alors que les suffètes carthaginois étaient au nombre de deux, élus pour une année. L'explication tient sans doute au fait que le titre de suffète n'avait à Makhtar plus de lien direct avec la fonction punique, le suffétat, pouvoir exécutif dans la constitution carthaginoise, qui faisait l'admiration d'Aristote.

- Des textes votifs néo-puniques mentionnent les ba'li de Makthar (= «citoyens de M.») qui remercient Baal Hammon d'avoir « entendu leur appel et de les avoir bénis » sans que l'on sache exactement à quelle occasion ce type de manifestation avait lieu.

- Vers 180 après J.C., la cité devient colonie romaine. L'inscription des pagi Tuscae et Gunzuzi découverte réemployée dans la place dite « agora numide » mentionne que Makthar était un centre de collecte des impôts. Elle date du II ${ }^{\mathrm{e}}$ siècle après J.C.

13 L'histoire de Makthar peut donc être partiellement reconstituée grâce aux documents épigraphiques qui y ont été découverts. Elle continue cependant d'interpeller les historiens ; la place de Makthar à l'époque pré-romaine notamment pose problème. Son statut de centre fiscal au II ${ }^{\mathrm{e}}$ après J.C. signifie-t-il pour autant que la ville jouait déjà le rôle de "capitale régionale » durant la période punique et numide comme le laissent croire les écrits de Picard, repris par de nombreux chercheurs ? L'inscription dite «la borne-limite " dans le Jbel Massouge mentionne un certain Wlb', chef des territoires TŠKT, et probablement neveu du roi en exercice, Micipsa*; cette découverte, conduit à penser que ce haut fonctionnaire siégeait en une ville qui pouvait revendiquer le statut de «capitale régionale ». Mais rien ne permet de conclure qu'il s'agissait de Makthar car il suffit de regarder la carte pour constater qu'à l'époque préromaine, et plus précisément au $\mathrm{II}^{\mathrm{e}}$ siècle avant J.C., les cités importantes capables de jouer ce rôle étaient nombreuses dans cette partie du Haut Tell. Cette identification, sans fondement précis, de Makthar comme lieu d'exercice de Wlb' pourrait provenir de ce que la fameuse borne-limite a été positionnée par G. C. Picard par rapport à Makthar.

\section{BIBLIOGRAPHIE}

CAMPS G., Aux origines de la Berbérie. Monuments et rites funéraires protohistoriques, Paris 1961.

CAMPS G., Corpus des poteries modelées retirées des monuments funéraires protohistoriques de l'Afrique du nord, Paris 1964.

СНАВОТ J.-В., Recueil des inscriptions libyques, Paris 1940. 
FAYOLLE V., La poterie modelée du Maghreb oriental, CNRS, Paris, 1992.

FÉVRIER J. G. \& FANTAR M. H., « Les nouvelles inscriptions monumentales néo-puniques de Mactar », Karthago 12, 1965, 43-59.

FÉVRIER J. G., « La grande inscription dédicatoire de Maktar », Sémitica, 6, 1956, 15-31.

FÉVRIER J. G., « La borne de Micipsa », Cahiers de Byrsa VII, 1957, 119-121.

GASCOU J., La politique municipale de l'empire romain en Afrique proconsulaire de Trajan à Septime Sévère, Paris, 1972, 147-151.

GHAKI M., « Le nouveau monument mégalithique de Makthar, rapport préliminaire », Reppal X, 1998, 63-72.

GHAKI M., « La céramique modelée du nouveau mégalithe », Reppal XI, 1999, 95-124.

LASSÈRE J.M., Ubique populus, Paris 1977.

LEGLAY M., Saturne Africain, Monuments, Paris, 1960.

LEPELLEY C., Les cités de l'Afrique romaine au Bas Empire, 2 tomes, Paris, 1979 et 1981, t. II, 289-295.

MANDOUZE A., Prosopographie chrétienne du Bas empire, Afrique, Paris, CNRS, 1982, p. 303-533.

M'CHAREK A., Aspects de l'évolution démographique et sociale à Mactaris aux IIéme et IIIéme siècles ap. J.C., Tunis, 1982.

M'CHAREK A., " De Zama à Kairouan : La Thusca et la Gamonia ", dans Frontières et limites géographiques de l'Afrique du Nord antique et médiévale, Hommage à P. Salama, Paris, Publications de la Sorbonne, 1999, p. 139-183.

мтімет A., « Atlas protohistorique de Tunisie, feuille de Mactar, 1/200 000 ème », Africa VII/VIII, 1982, 7-54.

mTimet A. \& M'chareK A., « Données nouvelles sur l'abandon d'un dolmen de Mactaris », Cahiers de Tunisie XXX, 1982, 5-18.

NICOLET C., Rome et la conquête du monde méditerranéen, vol. 2, Genèse d'un empire, chap. 2 : « Les guerres puniques ", 1978, p. 594-626.

PAUPHILET P., « Monument mégalithique de Maktar », Karthago IV, 1953, 49-83.

PICARD G. C., « Mactar », Bulletin économique et social, juillet 1954, $\mathrm{n}^{\circ} 90$.

PICARD G. C., « Civitas Mactaritana », Karthago VIII, 1957.

PICARD G. C., « Essai d'interprétation du sanctuaire de Hoter Miscar », BAC 18B, 1982 (1988), 17-20.

PICARD G. C., MAHJOUBI A., BESCHAOUCh A, « Pagus Tuscae et Gunzuzi », CRAI, 1963, 124-130.

PICARD G. C. et al., Recherches archéologiques franco-tunsiennes à Mactar, I, La maison de Venus, Rome, coll. EFR, 34, 1977.

PREVOT F., Recherches franco-tunisiennes à Mactar, V, Les inscriptions chrétiennes, Rome, coll. EFR, 1984.

SZNYCER M., «Quelques observations sur la grande inscription dédicatoire de Mactar », Sémitica $28,1972,25-36$.

SZNYCER M., « Antiquités et Epigraphie nord-sémitique », Annuaire de l'EPHE, IV ${ }^{\mathrm{e}}$ section, 1975 et 1977. 
SZNYCER M. \& NICOLET C., Rome et la conquête du monde méditerranéen, vol. 2, Genèse d'un empire, chap. $1:$ «Carthage et la civilisation punique », 1978, p. 545-593.

INDEX

Mots-clés : Antiquité, Carthage, Céramique, Epigraphie, Libyque, Monument funéraire, Onomastique, Punique, Romain, Rome, Tunisie, Ville 